\title{
CCDC26 rs4295627 polymorphism (8q24.21) and glioma risk: a meta-analysis
}

\author{
H.W. Lu, M. Huang, J.H. Wang, X.L. Sun and Y.Q. Ke \\ National Key Clinic Specialty, Neurosurgery Institute of Guangdong Province, \\ Guangdong Provincial Key Laboratory on Brain Function Repair and \\ Regeneration, Department of Neurosurgery, Zhujiang Hospital, \\ Southern Medical University, Guangzhou, China \\ Corresponding author: Y.Q. Ke \\ E-mail: luhongwei11@foxmail.com
}

Genet. Mol. Res. 14 (4): 12074-12084 (2015)

Received February 3, 2015

Accepted May 15, 2015

Published October 5, 2015

DOI http://dx.doi.org/10.4238/2015.October.5.20

\begin{abstract}
The association between the CCDC26 rs4295627 single nucleotide polymorphism (SNP) and the glioma risk has been studied previously, but these studies have yielded conflicting results. The aim of the present study is to analyze this association more vigorously, by means of a meta-analysis. A comprehensive literature search was performed in databases PubMed and EMBASE. Six articles including 12 case-control studies in English with 11,368 controls and 5891 cases were eligible for the meta-analysis. We conducted subgroup analyses by the source of controls, ethnicity, and country. Our meta-analysis revealed that the rs4295627 SNP was associated with the glioma risk in a heterozygote model (TG versus TT: odds ratio $=1.35,95 \%$ confidence interval $=1.26-1.45, \mathrm{P}=0.066)$. Moreover, our results suggested that the rs4295627 SNP was associated with a notably increased risk of glioma among Caucasians except for Swedes in 4 models (the homozygote model, recessive model, dominant model, and additive model). Nonetheless, in Sweden and China, the results showed no associations. No evidence of the publication bias was uncovered. Thus,
\end{abstract}


our meta-analysis suggests that the rs4295627 SNP is associated with an increased risk of glioma. Additional studies are needed to derive more precise conclusion.

Key words: Glioma; CCDC26; rs4295627; Polymorphism; Meta-analysis

\section{INTRODUCTION}

Gliomas are the most common tumor of the central nervous system in adults, accounting for more than $70 \%$ of all brain tumors, and among these, glioblastoma multiforme is the most frequent and malignant histological type (Ohgaki, 2009). Based on the cellular lineage, gliomas are classified as astrocytomas, oligodendrogliomas, oligoastrocytomas, and glioblastomas; these tumors can also be classified into 4 clinical grades: World Health Organization (WHO) grade I (pilocytic astrocytomas), WHO grade II (diffuse low-grade gliomas), WHO grade III (anaplastic gliomas), and WHO grade IV (glioblastoma multiforme) (Louis et al., 2007). The mechanisms of glioma carcinogenesis remain unclear. More and more studies suggest that various genetic alterations play a pivotal role in glioma susceptibility (Liu et al., 2010; Duncan and Yan, 2011).

The $C C D C 26$ gene is located in the chromosomal region 8q24.21. It is a retinoic acid modulator of differentiation and death (Yin et al., 2006). The rs4295627 single nucleotide polymorphism (SNP) is a $\mathrm{G} / \mathrm{T}$ variation mapped to intron 3 of $C C D C 26$. Genetic association studies, especially genome-wide association studies (GWAS), indicated that the rs4295627 SNP may contribute to the increased risk of glioma (Shete et al., 2009; Di Stefano et al., 2013), but the results are inconclusive (Egan et al., 2011; Li et al., 2012). To gain a better insight into the influence of rs 4295627 polymorphic variants on the risk of glioma, we performed a metaanalysis of all eligible case-control studies to obtain a more precise estimate of the association between the CCDC26 rs4295627 SNP and glioma risk.

\section{MATERIAL AND METHODS}

\section{The search for studies and selection}

A comprehensive literature search was performed in PubMed and EMBASE using the following search string: (CCDC26 or rs4295627) and (variant or polymorphism or variation) and (glioma or "brain tumor"). Additional studies were identified by hand when reviewing references in the found articles. We used the following inclusion criteria for our meta-analysis: 1) a case-control study evaluating the rs $4295627 \mathrm{SNP}$ in the CCDC26 gene, 2) a full-text article is available, 3 ) sufficient data for estimation of an odds ratio (OR) with a $95 \%$ confidence interval (CI), 4) genotype distribution of the control population must be in the Hardy-Weinberg equilibrium (HWE), and 5) no overlapping data. If some studies contained the same or overlapping data, only the largest study was included in the final analysis. Studies that were not related to human cancer research or did not contain sufficient data were excluded.

\section{Data extraction}

Three investigators independently extracted the data from all the eligible studies ac- 
cording to the criteria listed above. Any disagreement was resolved by discussion. The following data were collected from each study: the 1st author, publication year, country, racial origin (categorized as Asian, Caucasian, or of mixed descent), source of controls, genotyping method, numbers of cases and controls, genotype frequency of cases and controls, and the result of the HWE test.

\section{Statistical analysis}

A chi-square test in web-based software (http://ihg.gsf.de/cgi-bin/hw/hwa1.pl) was used to determine whether the observed frequencies of genotypes in controls conformed to the HWE ( $\mathrm{P}<0.05$ was assumed to denote statistical significance). The crude ORs and $95 \% \mathrm{CIs}$ in each case-control study were used to assess the strength of the associations between the glioma risk and the CCDC26 rs4295627 SNP. The pooled ORs were processed by means of the codominant model (GG versus TT; TG versus TT), dominant model (TG + GG versus $\mathrm{TT})$, recessive model ( $\mathrm{GG}$ versus $\mathrm{TT}+\mathrm{TG})$, and an additive model $(2 \mathrm{GG}+\mathrm{TG}$ versus $2 \mathrm{TT}+$ TG). Subgroup analyses were performed on the basis of ethnicity, country, and the source of controls. The heterogeneity assumption was tested by means of the Q-test, and the $\mathrm{I}^{2}$ statistic $\left(\mathrm{I}^{2}=100 \% \times\right.$ [Q - d.f.] / Q) was calculated to quantify the proportion of the total variation across the studies that was due to heterogeneity (Higgins et al., 2003). If the $P$ value was $<0.05$ in the Q-test and indicated the existence of heterogeneity among the studies, then the pooled OR estimate of each study was calculated by means of the random-effect model (DerSimonian and Laird, 1986). Otherwise, the fixed-effect model was used the Mantel-Haenszel method (Mantel and Haenszel, 1959). An estimate of a possible publication bias was calculated using the funnel plot, in which the standard error of $\log (\mathrm{OR})$ of each study was plotted against its $\log (\mathrm{OR})$. The funnel plot asymmetry was further evaluated by means of the Egger linear regression test $(\mathrm{P}<0.05$ was assumed to denote a significant publication bias) (Egger et al., 1997). All statistical analyses were performed in the STATA software (version 12; Stata Corporation, College Station, TX, USA).

\section{RESULTS}

\section{Extraction process and study characteristics}

A total of 6 articles (Shete et al., 2009; Schoemaker et al., 2010; Wang et al., 2011; Li et al., 2012; Di Stefano et al., 2013; Safaeian et al., 2013) including 12 case-control studies in English with 11,368 controls and 5891 cases were eligible for the meta-analysis. The main results of this meta-analysis are listed in Table 1 . These 12 studies included 11 studies of Caucasians and 1 study of Asians, 9 studies with a population-based control, and 3 studies with a hospital-based control. Figure 1 shows the study selection procedure.

\section{Meta-analysis results}

Table 2 lists the main results of the meta-analysis for the CCDC26 rs4295627 SNP. A significant association between this SNP and the glioma risk was observed when all eligible studies were pooled into the meta-analysis [GG versus TT: $\mathrm{OR}=1.75,95 \% \mathrm{CI}=1.27-2.40$; $\mathrm{TG}$ versus TT: $\mathrm{OR}=1.35,95 \% \mathrm{CI}=1.26-1.45$ (Figure 2); recessive model: $\mathrm{OR}=1.59,95 \% \mathrm{CI}=1.19-2.12$; dominant model: $\mathrm{OR}=1.37,95 \% \mathrm{CI}=1.22-1.55$; additive model: $\mathrm{OR}=1.33,95 \% \mathrm{CI}=1.17-1.50]$. 


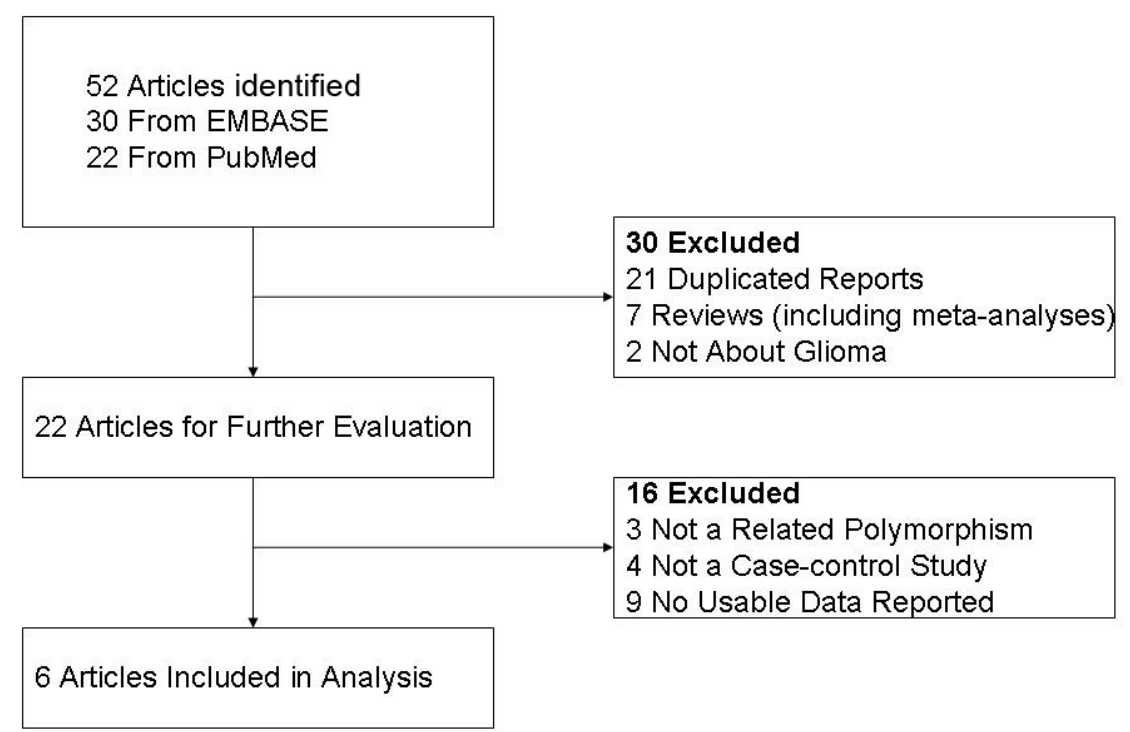

Figure 1. Flow diagram of the studies included.

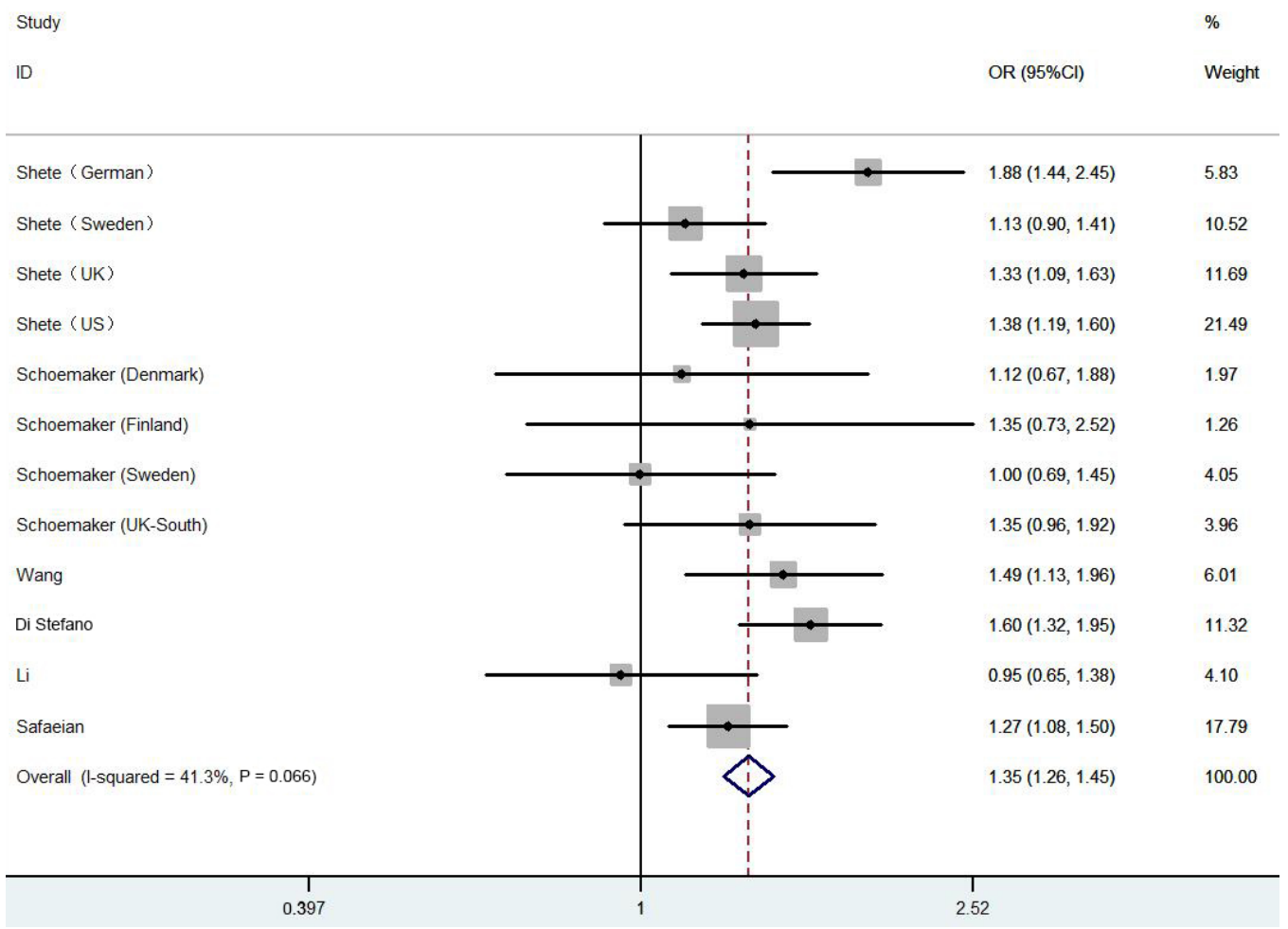

Figure 2. Forest plots from the meta-analysis of CCDC26 rs4295627 polymorphism and the risk of glioma observed in total in the heterozygote model (TG vs TT). 


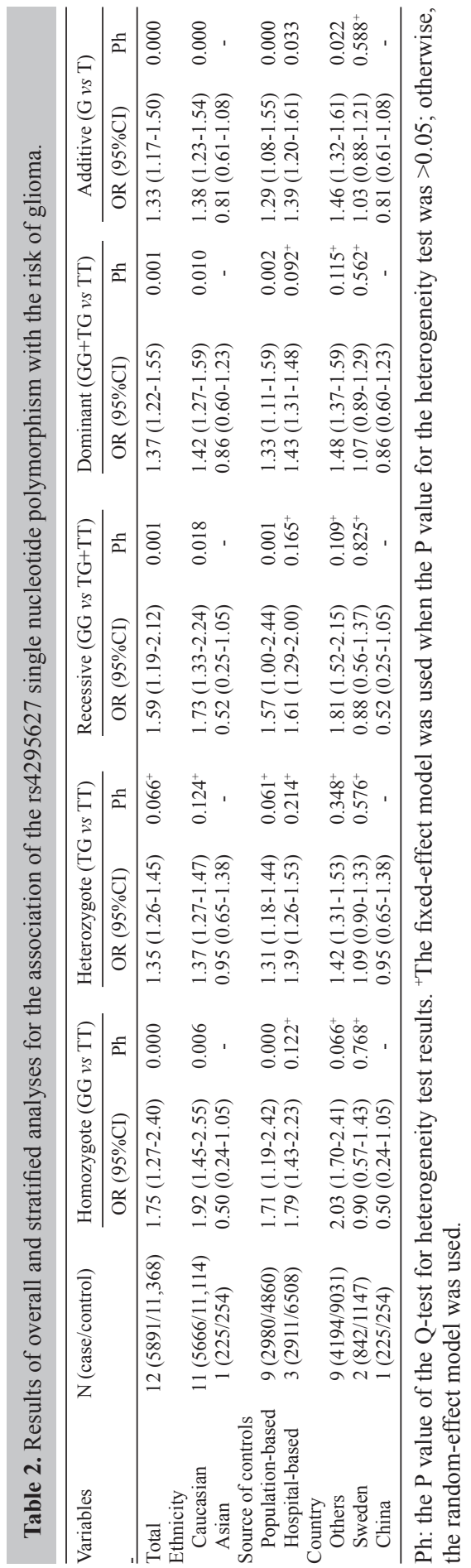


As for ethnicity, our results showed that the rs4295627 SNP was associated with an increased risk of glioma among Caucasians (GG versus TT:OR $=1.92,95 \% \mathrm{CI}=1.45$ 2.55; $\mathrm{TG}$ versus TT: $\mathrm{OR}=1.37,95 \% \mathrm{CI}=1.27-1.47$; recessive model: $\mathrm{OR}=1.73,95 \% \mathrm{CI}$ $=1.33-2.24$; dominant model: $\mathrm{OR}=1.42,95 \% \mathrm{CI}=1.27-1.59$; additive model: $\mathrm{OR}=1.38$, $95 \% \mathrm{CI}=1.23-1.54$ ) but not among Asians. When we stratified the data by the source of controls, we found an increased risk of glioma in hospital-based studies (GG versus TT: $\mathrm{OR}=1.79,95 \% \mathrm{CI}=1.43-2.23$; $\mathrm{TG}$ versus $\mathrm{TT}: \mathrm{OR}=1.39,95 \% \mathrm{CI}=1.26-1.53$; recessive model: $\mathrm{OR}=1.61,95 \% \mathrm{CI}=1.29-2.00$; dominant model: $\mathrm{OR}=1.43,95 \% \mathrm{CI}=1.31-1.48$; additive model: $\mathrm{OR}=1.39,95 \% \mathrm{CI}=1.20-1.61$ ) but not in population-based studies. In agreement with another study (Shete et al., 2009), there was evidence of between-study heterogeneity ascribable to the association's being modest in the Swedish series. Nevertheless, we subdivided Caucasians into 2 subgroups: Swedes and others. Thus, the data were subdivided by country into 3 subgroups: China, Sweden, and others.

Our results suggested that the rs4295627 SNP was associated with a notably increased risk of glioma in countries other than Sweden and China (GG versus TT: OR $=2.03,95 \% \mathrm{CI}=$ 1.70-2.41; TG versus TT: $\mathrm{OR}=1.42,95 \% \mathrm{CI}=1.31-1.53$; recessive model: $\mathrm{OR}=1.81,95 \% \mathrm{CI}$ $=1.52-2.15$; dominant model: $\mathrm{OR}=1.48,95 \% \mathrm{CI}=1.37-1.59$; additive model: $\mathrm{OR}=1.46$, $95 \% \mathrm{CI}=1.32-1.61$; Table 2). In contrast, in Sweden and China, the results seemed to be the opposite (Figure 3). All results of the genetic models are listed in Table 2.

\section{Test of heterogeneity}

This test showed that there was significant between-study heterogeneity in terms of the rs4295627 SNP in 4 models (homozygote model, recessive model, dominant model, and additive model; Table 2). When subgroup analyses were based on ethnicity and the source of controls, the heterogeneity still appeared. In contrast, when we subdivided the results by country, the heterogeneity appeared only in the "Others" subgroup in the additive model $(\mathrm{P}=$ 0.022 ; Table 2).

\section{Publication bias}

Both Begg's funnel plot and the Egger test were performed to assess the publication bias in this literature. The polymorphism showed consistent results, indicating the absence of the publication bias. The shapes of the funnel plot did not indicate any evidence of obvious asymmetry in the heterozygote model (Figure 4), and the results of the Egger test were suggestive of the absence of the publication bias (GG versus TT: $\mathrm{P}=0.72$, TG versus TT: $\mathrm{P}=0.44$; recessive model: $\mathrm{P}=0.644$; dominant model: $\mathrm{P}=0.653$, and additive model: $\mathrm{P}=0.872$ ).

\section{DISCUSSION}

SNPs are known to be associated with many diseases (Wu and Jin, 2013). A GWAS is a powerful research strategy that uses SNPs as markers to identify susceptibility genes of many complex diseases (Porcu et al., 2013). Many meta-analyses have already been con- 


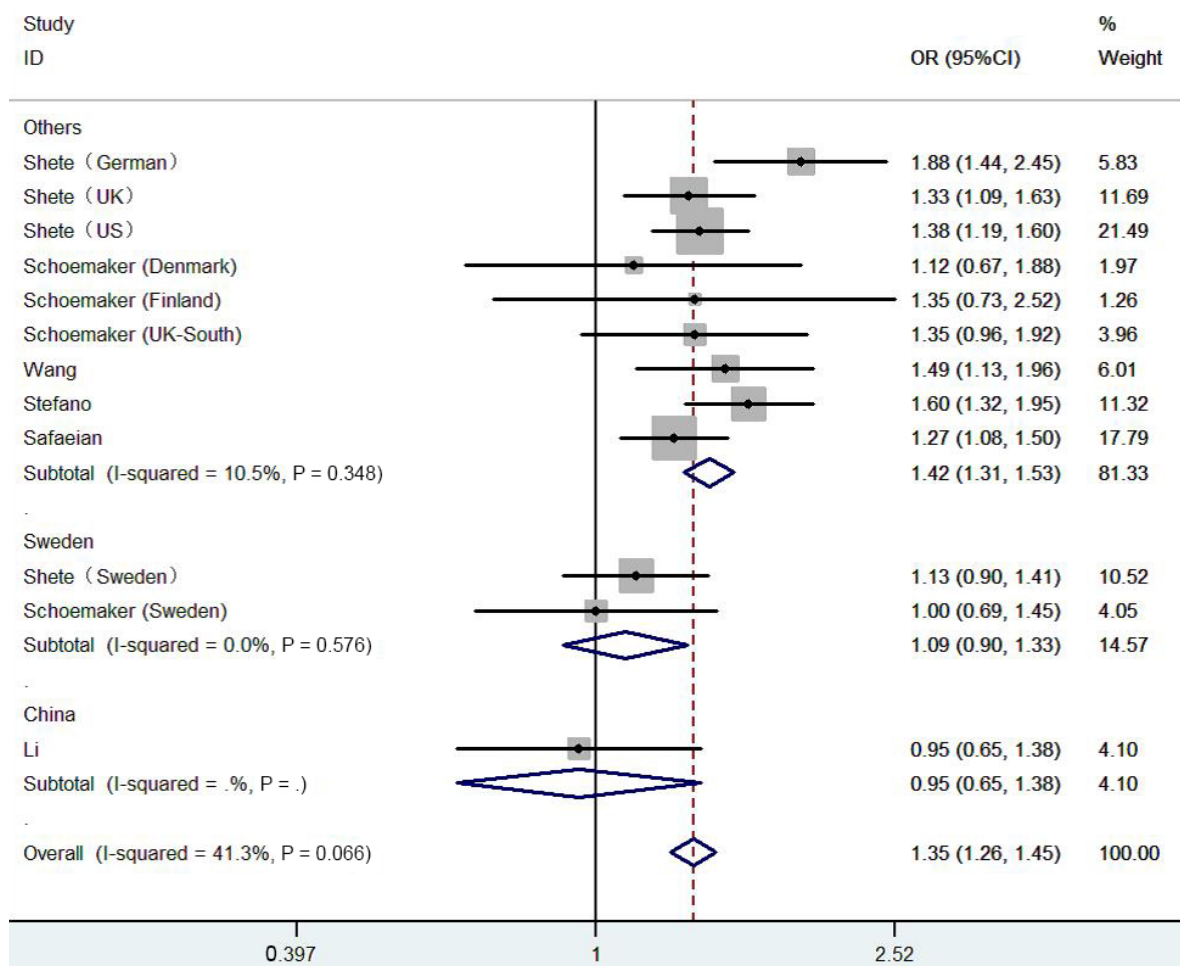

Figure 3. Forest plots from the meta-analysis of CCDC 26 rs 4295627 polymorphism and the risk of glioma stratified by country in heterozygote model (TG vs TT).

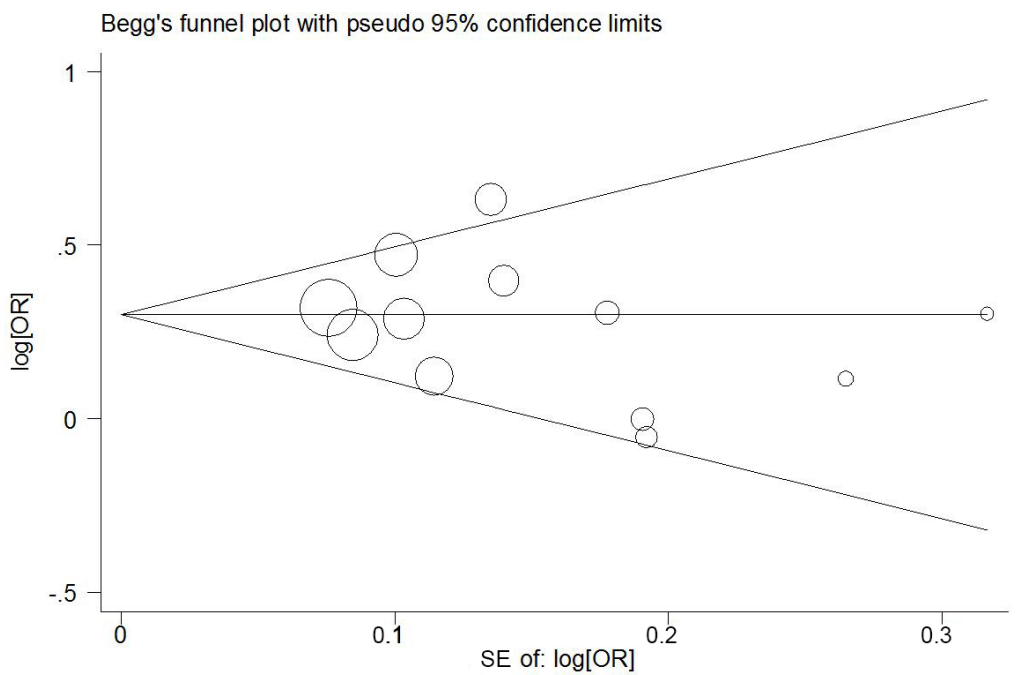

Figure 4. Begg's funnel plots of the rs4295627 polymorphism and glioma risk for publication bias test in the heterozygote model (TG vs TT). 
ducted to explore the association between an SNP and the risk of glioma (Jiang et al., 2013; Gao et al., 2014; Zhou et al., 2014). Other studies have shown that CCDC26 is a retinoic acid modulator of differentiation and death (Jiang et al., 2008). Retinoic acid induces caspase 8 transcription through phosphorylation of the cAMP response element-binding protein and enhances apoptosis in response to death stimuli in neuroblastoma cells and in glioblastoma cells with downregulation of the telomerase activity (Jiang et al., 2008). The rs4295627 SNP is a $\mathrm{G} / \mathrm{T}$ variation mapped to intron 3 of the CCDC26 gene. Various studies have suggested that the rs 4295627 SNP is significantly associated with an increased risk of glioma. For example, one study showed this result via analysis of 5 glioma susceptibility loci, including rs 4295627 (Shete et al., 2009). However, the individual studies might have been underpowered and thus unable to detect the overall effect of SNPs on the risk of glioma. In order to resolve this issue, we performed a meta-analysis to accomplish the most comprehensive assessment of the association between the rs4295627 SNP and glioma risk.

In this meta-analysis, which includes 5891 cases and 11,368 controls from 12 studies (6 articles), we ascertained that the rs4295627 SNP is significantly associated with an increased glioma risk. As for ethnicity, rs4295627 seems to be associated with an increased risk of glioma among Caucasians in the heterozygote model, but we could not obtain the same conclusion in the other genetic models because of the significant heterogeneity. As for Asians, there was only one study; thus, we could not draw a conclusion.

Researchers should pay more attention to the risk of glioma among Asians. Shete et al. (2009) mentioned that a study from Sweden caused between-study heterogeneity in their analysis. Accordingly, we tried to subdivide Caucasians into 2 subgroups: Sweden and "Others". In the "Others" group, our results revealed a significantly increased risk of glioma in the homozygote, heterozygote, recessive, and dominant models. In the Sweden group, however, we concluded that rs4295627 is not associated with an increased risk of glioma in Sweden. We do not know the reason for this discrepancy. Perhaps, among the studies, there is some variation in the design, sample size, inclusion criteria, ethnicity, or other parameters (Huang et al., 2014). Because there are only two studies in the Sweden group, more studies should be conducted to confirm our findings.

As for the source of controls, a significantly increased risk was observed in the population-based group in the heterozygote model and in the hospital-based group in the homozygote, heterozygote, recessive, and dominant models. The population-based controls might be more effective at reducing biases because such controls can be representative of the general population (Gao et al., 2014). In our results, however, there is significant heterogeneity in the majority of the genetic models within the population-based group. Other factors may contribute to this phenomenon.

There are possible limitations to our meta-analysis. First, the number of the studies analyzed is not sufficiently large for a comprehensive study, especially for analyses of subgroups. Only one study on Asians is present in this meta-analysis. More studies are needed to determine whether the SNP in question affects the risk of glioma in various ethnic groups. Second, our results are based on unadjusted estimates, whereas a more accurate OR can be obtained by adjusting the raw OR for age, gender, drinking, smoking, and other factors that are associated with the cancer risk (Jiang et al., 2013). Third, because more detailed individual information on the gene-gene and gene-environment interactions was unavailable, we were unable to conduct a more precise analysis. Fourth, overall, there is significant between-study heterogeneity, which may reduce the quality of evidence of this meta-analysis. On the other 
hand, when the data are subdivided by country, the heterogeneity can be ignored.

Our meta-analysis has some advantages. First, the well-designed selection methods increased the statistical power of our meta-analysis. Second, the results do not show any evidence of the publication bias. Third, the subgroup analysis by country decreased the heterogeneity and helped us to draw a more accurate conclusion. Fourth, all genotypes of the controls are compliant with the HWE $(\mathrm{P}>0.05)$.

In conclusion, this meta-analysis suggests that the rs $4295627 \mathrm{SNP}$ in the CCDC26 gene is significantly associated with an increased glioma risk. There is an increased risk in most Caucasians (except Swedes). In China and Sweden, however, rs4295627 is not associated with an increased risk of glioma. Additional studies are needed to draw a more precise conclusion.

\section{Conflicts of interest}

The authors declare no conflict of interest.

\section{ACKNOWLEDGMENTS}

Research supported in part by the National Natural Science Foundation of China [grants \#81272806 (to Y.Q. Ke) and \#81302199 (to X.L. Sun)], the Natural Science Foundation of Guangdong Province, China (grant \#S2012010009088 to Y.Q. Ke), and the Medical Scientific Research Foundation of Guangdong Province, China (grant \#B2013246 to X.L. Sun).

\section{REFERENCES}

DerSimonian R and Laird N (1986). Meta-analysis in clinical trials. Control. Clin. Trials 7: 177-188.

Di Stefano AL, Enciso-Mora V, Marie Y, Desestret V, et al. (2013). Association between glioma susceptibility loci and tumour pathology defines specific molecular etiologies. Neuro-Oncol. 15: 542-547.

Duncan CG and Yan H (2011). Genomic alterations and the pathogenesis of glioblastoma. Cell Cycle 10: 1174-1175.

Egan KM, Thompson RC, Nabors LB, Olson JJ, et al. (2011). Cancer susceptibility variants and the risk of adult glioma in a US case-control study. J. Neurooncol. 104: 535-542.

Egger M, Davey SG, Schneider M and Minder C (1997). Bias in meta-analysis detected by a simple, graphical test. B.M.J. 315: 629-634.

Gao X, Mi Y, Yan A, Sha B, et al. (2014). The PHLDB1 rs498872 (11q23.3) polymorphism and glioma risk: A metaanalysis. Asia Pac. J. Clin. Oncol. doi: 10.1111/ajco.12211.

Higgins JP, Thompson SG, Deeks JJ and Altman DG (2003). Measuring inconsistency in meta-analyses. B.M.J. 327 : $557-560$.

Huang LM, Shi X, Yan DF, Zheng M, et al. (2014). Association Between ERCC2 Polymorphisms and Glioma Risk: a Meta-analysis. Asian Pac. J. Cancer Prev. 15: 4417-4422.

Jiang L, Fang X, Bao Y, Zhou J, et al. (2013). Association between the XRCC1 Polymorphisms and Glioma Risk: A MetaAnalysis of Case-Control Studies. PLoS One 8: e55597.

Jiang M, Zhu K, Grenet J and Lahti JM (2008). Retinoic acid induces caspase-8 transcription via phospho-CREB and increases apoptotic responses to death stimuli in neuroblastoma cells. B.B.A.-Mol. Cell. Res. 1783: 1055-1067.

Li S, Jin T, Zhang J, Lou H, et al. (2012). Polymorphisms of TREH, IL4R and CCDC26 genes associated with risk of glioma. Cancer Epidemiol. 36: 283-287.

Liu Y, Shete S, Hosking FJ, Robertson LB, et al. (2010). New insights into susceptibility to glioma. Arch. Neurol. 67: 275-278.

Louis DN, Ohgaki H, Wiestler OD, Cavenee WK, et al. (2007). The 2007 WHO Classification of Tumours of the Central Nervous System. Acta Neuropathol. 114: 97-109.

Mantel N and Haenszel W (1959). Statistical aspects of the analysis of data from retrospective studies of disease. J. Natl. Cancer Inst. 22: 719-748. 
Ohgaki H (2009). Epidemiology of brain tumors. Methods Mol. Biol. 472: 323-342.

Porcu E, Sanna S, Fuchsberger C and Fritsche LG (2013). Genotype imputation in genome-wide association studies. Curr. Protoc. Hum. Genet. Unit. 1: 25.

Safaeian M, Rajaraman P, Hartge P, Yeager M, et al. (2013). Joint effects between five identified risk variants, allergy, and autoimmune conditions on glioma risk. Cancer Cause Control 24: 1885-1891.

Schoemaker MJ, Robertson L, Wigertz A, Jones ME, et al. (2010). Interaction Between 5 Genetic Variants and Allergy in Glioma Risk. Am. J. Epidemiol. 171: 1165-1173.

Shete S, Hosking FJ, Robertson LB, Dobbins SE, et al. (2009). Genome-wide association study identifies five susceptibility loci for glioma. Nat. Genet. 41: 899-904.

Wang SS, Hartge P, Yeager M, Carreon T, et al. (2011). Joint Associations Between Genetic Variants and Reproductive Factors in Glioma Risk Among Women. Am. J. Epidemiol. 174: 901-908.

Wu J and Jiang R (2013). Prediction of deleterious nonsynonymous single-nucleotide polymorphism for human diseases. Sci. World J. 2013: 675851.

Yin W, Rossin A, Clifford JL and Gronemeyer H (2006). Co-resistance to retinoic acid and TRAIL by insertion mutagenesis into RAM. Oncogene 25: 3735-3744.

Zhou P, Wei L, Xia X, Shao N, et al. (2014). Association between telomerase reverse transcriptase rs 2736100 polymorphism and risk of glioma. J. Surg. Res. 191: 156-160. 